\title{
INDENTATION PRESSURE OF A SMOOTH CIRCULAR PUNCH*
}

\section{Br}

E. LEVIN

National Bureau of Standards, Los Angeles, Calif.

Abstract. A smooth, flat, rigid punch under increasing normal load presses against a half space of a perfectly plastic material which obeys Tresca's yield criterion. An admissible velocity field is constructed for an arbitrary smooth punch hence, for any particular case, a limit design theorem of Drucker, Prager and Greenberg may be used to compute an upper bound for the punch indentation pressure. A lower bound for any convex area of indentation has been given by Shield and Drucker. The results of the present paper are used to compute an upper bound for a punch with circular cross section. It is conjectured that this is an upper bound for an arbitrary punch.

1. Introduction. The exact solutions of any but the most trivial problems involving plastic deformation have thus far been impossible to obtain. Consequently the limit design theorems of Drucker, Prager and Greenberg [1] which provide upper and lower bounds on the collapse load have become the most powerful tools available for the analysis of such problems. In the present paper this technique is used to obtain an upper bound on the punch pressure at the moment of impending plastic indentation.

Consider a flat rigid punch subjected to increasing normal load and pressing against a half-space (Fig. 1). The half-space is assumed to be of an elastic-perfectly plastic

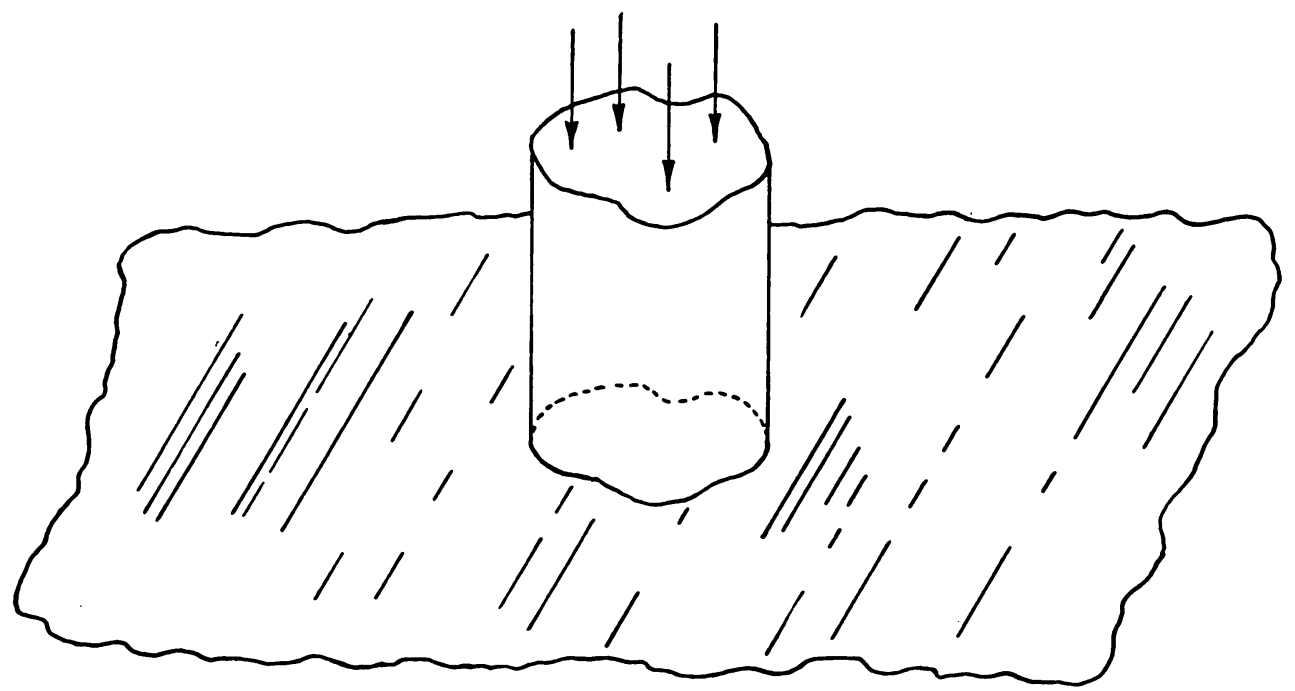

Fig. 1. Punch on halt space.

material which obeys Tresca's [2] yield criterion of constant maximum shearing stress, $k$, during plastic deformation. The pressure is gradually increased until indentation due to plastic flow of the material is impending. Geometry changes up to this point are

${ }^{*}$ Received April 10, 1954. The preparation of this paper was sponsored in part by the Office of Naval Research, USN. 
presumably small and are neglected. The actual load applied to the end of the punch may be concentrated or distributed but it is assumed that over the contact area there is a uniformly distributed normal pressure.

According to the Drucker-Prager-Greenberg theorem an upper bound on this indentation pressure, $p$, may be obtained by constructing a kinematically admissible velocity field. Such a field must satisfy the following conditions:

(a) it must accommodate the assumed downward motion of the punch;

(b) it must be incompressible in the sense that

$$
\epsilon_{1}+\epsilon_{2}+\epsilon_{3}=0,
$$

where $\epsilon_{1}, \epsilon_{2}, \epsilon_{3}$ are three orthogonal strain rates;

$$
\int_{S} p v d S \geq \int_{V} 2 k \max |\epsilon| d V+\int_{S_{D}} k \Delta v d S_{D},
$$

where $S$ is the contact surface between the punch and the material, $V$ is the volume of the deforming material, $S_{D}$ represents surfaces across which the tangential velocity is discontinuous, $\Delta v$ is the magnitude of the relative change in velocity across $S_{D}, \max |\epsilon|$ represents the absolutely largest principal strain rate component.

Actually the third condition is used to compute $p$ when a velocity field satisfying the first two conditions has been found. If this velocity field involves slip between the punch and the material, it is necessary to make the further restriction that the punch be smooth.

2. General case. In this section an admissible velocity field is constructed for an arbitrary punch. Since the velocity field involves slip between the punch and the material it is assumed that the punch is smooth. The only restriction on the shape of the punch is that its cross-section be star-shaped (Fig. 2). Thus the boundary of the cross-section

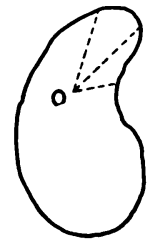

(a)

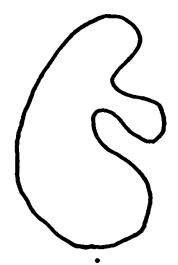

(b)

Fig. 2. (a) Admissible cross section

(b) Inadmissible cross section.

can be described in polar coordinates by $r=d(\theta)$ where $d$ is a continuous single-valued function. This is a very weak restriction and admits, for example, any punch of convex cross section.

For a given punch a suitable origin is selected and a cylindrical coordinate system is established at this point. In each section $\theta$ an assumption is made as to the general shape of the velocity field (Fig. 3). The exact form is then determined so as to satisfy the requirements for a kinematically admissible velocity field.

In region $A$ assume

$$
V_{\theta}=0, \quad V_{r}=V_{z}=F .
$$


Then to satisfy incompressibility

A solution is

$$
\frac{\partial F}{\partial r}+\frac{F}{r}+\frac{\partial F}{\partial z}=0
$$

$$
F=\phi(r-z) / r .
$$

To accommodate the assumed downward motion of the punch $\left[V_{z}\right]_{z=0}=v$. Hence

$$
F=v(r-z) / r \text {. }
$$

In each of the remaining two regions a similar assumption as to the general shape

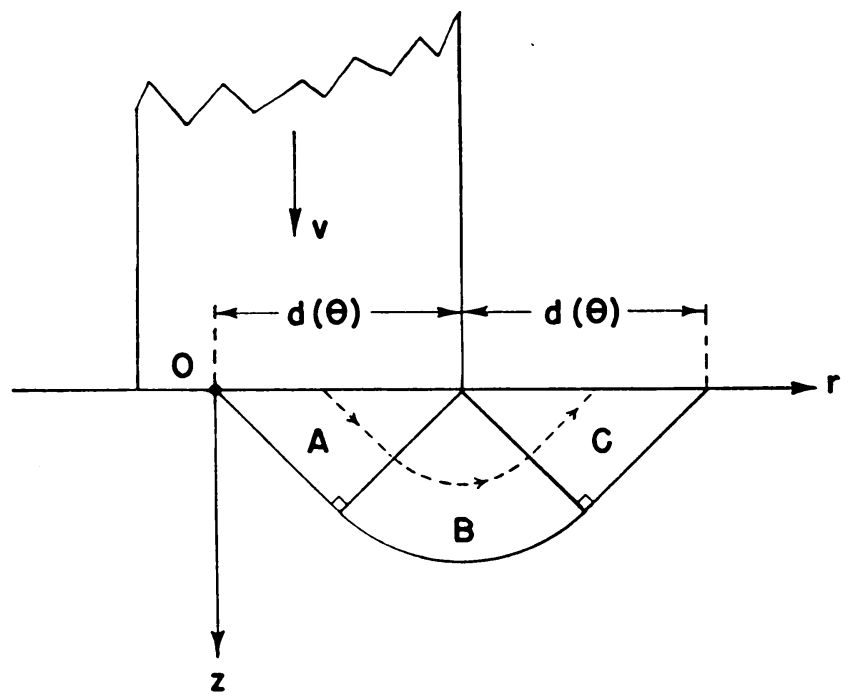

FIG. 3. Velocity Field.

of the velocity field is made in accord with Fig. 3. The exact form is then determined so as to satisfy the incompressibility condition. The results are presented in Table 1.

TABLE 1

\begin{tabular}{|c|c|c|c|}
\hline Region & $V_{r}$ & $V_{z}$ & $V_{\theta}$ \\
\hline$A$ & $F$ & $F$ & 0 \\
$B$ & $z G$ & $(d-r) G$ & 0 \\
$C$ & $H$ & $-H$ & 0 \\
\hline
\end{tabular}

where $\quad F=v(r-z) / r, \quad G=\frac{2^{1 / 2} v\left[d-2^{1 / 2}\left\{(d-r)^{2}+z^{2}\right\}^{1 / 2}\right]}{r\left\{(d-r)^{2}+z^{2}\right\}^{1 / 2}}, \quad H=v(2 d-r-z) / r$.

As well as satisfying the incompressibility condition, it can be verified that this field is continuous between the three regions and vanishes on the boundaries $r=z$, 
$\left[(d-r)^{2}+z^{2}\right]^{1 / 2}=d / 2^{1 / 2}$ and $r+z=2 d$. Hence the last integral in Inequality 2 vanishes and an upper bound on the indentation pressure is furnished by any pressure, $p$, satisfying

$$
\frac{p v}{2} \int_{0}^{2 \pi}[d(\theta)]^{2} d \theta \geq 2 k \int_{V} \max |\epsilon| d V .
$$

3. Circular punch. For the particular case of a punch with circular cross section, $\boldsymbol{p}(\theta)$ is a constant which will be taken as unity. The strain rates appear in Table 2 .

TABLE 2

\begin{tabular}{|c|c|c|c|}
\hline & $A$ & $B$ & $C$ \\
\hline$\epsilon_{r r}$ & $\frac{v z}{r^{2}}$ & $\frac{2 v z}{r^{2}}-\frac{2^{1 / 2} v z\left(2 r^{2}-3 r+1+z^{2}\right)}{r^{2}\left\{(1-r)^{2}+z^{2}\right\}^{3 / 2}}$ & $-\frac{v(2-z)}{r^{2}}$ \\
\hline$\epsilon_{\theta \theta}$ & $\frac{v(r-z)}{r^{2}}$ & $\frac{2^{1 / 2} v z}{r^{2}\left\{(1-r)^{2}+z^{2}\right\}^{1 / 2}}-\frac{2 v z}{r^{2}}$ & $\frac{v(2-r-z)}{r^{2}}$ \\
\hline$\epsilon_{3 z}$ & $-\frac{v}{r}$ & $-\frac{2^{1 / 2} v z(1-r)}{\left.r\left\{(1-r)^{2}+z^{2}\right)\right\}^{3 / 2}}$ & $\frac{v}{r}$ \\
\hline$\epsilon_{r z}$ & $\frac{v(z-r)}{2 i^{2}}$ & $\frac{v(1-r)}{r^{2}}+\frac{2^{1 / 2} v(1-r)^{2}}{r\left\{(1-r)^{2}+z^{2}\right\}^{3 / 2}}-\frac{2^{1 / 2} v}{2 r^{2}\left\{(1-r)^{2}+z^{2}\right\}^{1 / 2}}$ & $\frac{v(2-r-z)}{2 r^{2}}$ \\
\hline
\end{tabular}

The principal strain rates are

$$
\begin{aligned}
& \epsilon_{1}=\epsilon_{\theta \theta}, \\
& \epsilon_{2}=\frac{1}{2}\left[-\epsilon_{\theta \theta}+\left(\epsilon_{\theta \theta}^{2}+4 \epsilon_{r z}^{2}-4 \epsilon_{r r} \epsilon_{z z}\right)^{1 / 2}\right], \\
& \epsilon_{3}=\frac{1}{2}\left[-\epsilon_{\theta \theta}-\left(\epsilon_{\theta \theta}^{2}+4 \epsilon_{r z}^{2}-4 \epsilon_{r r} \epsilon_{z z}\right)^{1 / 2}\right] .
\end{aligned}
$$

Since $\epsilon_{\theta \theta} \geq 0$ in each region, the absolutely largest principal strain rate will be $\epsilon_{3}$ provided $4 \epsilon_{r z}^{2}-4 \epsilon_{r r} \epsilon_{s z} \geq 0$. This is easily verified in regions $A$ and $C$. In region $B$ it is found that

$$
\begin{aligned}
4 \epsilon_{r z}^{2}-4 \epsilon_{r r} \epsilon_{z z} & =\left[z \frac{\partial G}{\partial z}+(1-r) \frac{\partial G}{\partial r}\right]^{2}-4 z(1-r) \frac{\partial G}{\partial r} \frac{\partial G}{\partial z} \\
& =\left[z \frac{\partial G}{\partial z}-(1-r) \frac{\partial G}{\partial r}\right]^{2} \geq 0 .
\end{aligned}
$$

Hence in all three regions

$$
\operatorname{Max}|\epsilon|=\left|\epsilon_{3}\right|=\frac{1}{2}\left[\epsilon_{\theta \theta}+\left(\epsilon_{\theta \theta}^{2}+4 \epsilon_{r z}^{2}-4 \epsilon_{r r} \epsilon_{z z}\right)^{1 / 2}\right] .
$$

An upper bound is obtained as any value $p$ satisfying

$$
\frac{p v}{2} 2 \pi \geq 2 k \cdot 2 \pi \int_{A, B, C}\left|\epsilon_{3}\right| r d r d z .
$$


A portion of this integration was performed numerically. It was found that $5.84 k$ is an upper bound on the indentation pressure.

4. Discussion and conclusions. The assumed shape of the velocity field is patterned after the field constructed by Hill [3] for the two dimensional punch. Due to symmetry this field is most easily applied to a circular punch. For other cross-sections the general formulation given in Sec. 2 would involve considerable labour and indeed might not yield the sharpest upper bound.

For the two dimensional punch it is known that the indentation pressure is $(2+\pi) k$. In a recent paper by Shield and Drucker [4] on punches with rectangular cross section, a lower bound of $5 k$ was obtained for any convex area of indentation. Upper bounds varied between $(2+\pi) k$ for a very long rectangle (which agrees with the two dimensional solution) and $5.71 k$ for a square.

It may be noted that the results of Shield and Drucker indicate that the upper bound increases as the ratio of cross-sectional area to perimeter increases. Since the circular cross-section has the greatest area for a given perimeter, the upper bound of 5.84k for the circle appears quite reasonable as compared with the upper bound of $5.71 k$ for the square. Furthermore, it is conjectured that this value for the circle is an upper bound for an arbitrary punch. Combining the results of Shield and Drucker with the results and conjecture of the present paper indicates that the indentation pressure for any smooth punch with convex cross-section lies between $5 k$ and $5.84 k$.

\section{BibLIOGRAPHY}

1. D. C. Drucker, W. Prager and H. J. Greenberg, Extended limit design theorems for continuous media, Quart. Appl. Math. 9, 381-389 (1952)

2. H. Tresca, Mémoire sur l'écoulement des corps solides, Mémoires presentés par divers Savants 18, 733-799 (1868)

3. R. Hill, The plastic yielding of notched bars under tension, Quart. J. Mech. and Appl. Math. 2, 40-52 (1949)

4. R. T. Shield and D. C. Drucker, The application of limit analysis to punch indentation problems, J. Appl. Mech. 20, 453-460 (1953) 